\title{
Effect of Inflammation on the Relationship of Pulse Pressure and Mortality in Haemodialysis
}

\author{
Debasish Banerjee $^{a} \quad$ Allan J. Collins ${ }^{b} \quad$ Charles A. Herzog ${ }^{c}$ \\ ${ }^{a}$ Renal and Transplantation Unit, St. George's Hospital NHS Trust, London, UK; ${ }^{b}$ United States \\ Renal Data System, Minneapolis Medical Research Foundation, and ${ }^{\complement}$ Cardiovascular Special \\ Studies Center, United States Renal Data System, Minneapolis Medical Research Foundation, \\ Minneapolis, Minn., USA
}

\section{Key Words}

Haemodialysis $\cdot$ Inflammation $\cdot$ Mortality $\cdot$ Pulse pressure

\begin{abstract}
Background/Aim: The effect of hypertension on mortality in haemodialysis patients is controversial and can be confounded by non-traditional risk factors like systemic inflammation. This study examined the effect of systolic blood pressure (SBP), diastolic blood pressure (DBP), and pulse pressure (PP) on mortality in haemodialysis patients, separately with and without markers of systemic inflammation. Methods: Data from the United States Renal Data System were analysed for 9,862 patients receiving haemodialysis on December 31, 1993, followed through May 2005. Results: In Cox regression analysis, increased age, diabetes, low albumin, high white blood count, low cholesterol, low haemoglobin, high phosphate, low DBP, and cardiovascular comorbidity were associated with high mortality, but SBP was not. Elevated PP adjusted for SBP, age, diabetes, haemoglobin, albumin, cholesterol, calcium, phosphate, parathyroid hormone, and white blood count was associated with higher mortality [adjusted hazard ratio, PP 1.006 (95\% confidence interval, Cl, 1.002-1.010); SBP 0.993 (95\% Cl 0.990-0.996)]. In dual models, PP adjusted for SBP then DBP was associated with higher mortality [PP 1.029 (95\% Cl 1.027-1.032); SBP 0.981 (95\% Cl 0.979-0.983); PP 1.010 (95\% Cl 1.008-1.011); DBP 0.981 (95\% Cl 0.979-0.983)].
\end{abstract}

The interpretation and reporting of these data are the responsibility of the authors and in no way should be seen as an official policy or interpretation of the US government. 
Increasing PP deciles $>70 \mathrm{~mm} \mathrm{Hg}$ were associated with increasing mortality in the absence of markers of systemic inflammation (white blood count $>10 \times 10^{9} / \mathrm{l}$, albumin $<3.5 \mathrm{~g} / \mathrm{dl}$, diabetes), but not in their presence. Conclusion: PP was a better indicator of adverse outcome than DBP or SBP. Inflammation-associated injury may mask the effect of PP on mortality in haemodialysis patients.

Copyright $\odot 2011$ S. Karger AG, Basel

\section{Introduction}

Mortality in patients on maintenance haemodialysis is higher than in the general population across all age groups, with cardiovascular cause of death in about $50 \%$ [1]. Thus, obvious interest is directed towards traditional cardiovascular risk factors. Hypertension, being the most prevalent and potentially modifiable traditional cardiovascular risk factor, attracts further attention.

However, the relationship of mortality with hypertension has been controversial. Counterintuitively, in earlier studies a higher systolic blood pressure (SBP) was demonstrated to be protective. An SBP $<120 \mathrm{~mm} \mathrm{Hg}$ has been shown to be associated with increased mortality. Interestingly, the inverse relationship of SBP and mortality persisted even after adjustment for cardiovascular risk factors, hinting perhaps at the possibility of an independent strong effect of non-traditional risk factors [2].

Pulse pressure (PP) has been proven to be a better predictor of adverse outcome on haemodialysis than diastolic blood pressure (DBP) or even SBP [3, 4]. PP correlates with central arterial stiffness, which has adverse effects on the heart. The stiffness of the central arteries increases the cardiac afterload and decreases coronary perfusion [2].

Systemic inflammation, a non-traditional risk factor, is increasingly recognised as a powerful contributor to adverse outcomes in patients on maintenance haemodialysis [5]. The subgroup of haemodialysis patients with elevated markers of systemic inflammation tend to do poorly. The mechanism of this clinical phenomenon is largely unknown. No study has examined the effects of inflammation on the relationship of hypertension with mortality in haemodialysis patients.

The aim of this study was to explore the relationships of SBP, DBP, and PP with all-cause mortality in maintenance haemodialysis patients.

\section{Methods}

Data from the United States Renal Data System (USRDS) Dialysis Mortality and Morbidity Study (DMMS) Waves 3 and 4 were analysed retrospectively; 9,862 patients undergoing maintenance haemodialysis in the United States in December 1993 were included in the study. Baseline demographic information and clinical and laboratory parameters were collected in December 1993. Follow-up data on mortality were available by linking the DMMS data set to the USRDS database. Patients were followed till 2005. This retrospective cohort was managed very differently from the present patients on maintenance haemodialysis in 2011 regarding the use of angiotensin-converting enzymes, angiotensin receptor blockers, newer phosphate binders, statins, and aggressive cardiovascular screening.

A mean of three blood pressure readings in the last week of December 1993 was used for analysis. Relevant clinical variables at baseline were tested for their relationships with mortality. Variables associated with systemic inflammation, namely diabetes, white blood cell count, and low albumin, were included in the model. These markers of inflammation were 
Table 1. Clinical characteristics of the population

\begin{tabular}{lc}
\hline Variable & $\begin{array}{c}\text { Mean } \pm \text { SD } \\
\text { or percentage }\end{array}$ \\
\hline Age, years & $59 \pm 16$ \\
Males & 51 \\
Diabetes & 48 \\
Haemoglobin, g/dl & $10.1 \pm 2.3$ \\
Albumin, g/dl & $3.76 \pm 0.45$ \\
Cholesterol, mg/dl & $176 \pm 47$ \\
White blood count, $\times 10^{9} / 1$ & $7.9 \pm 5.7$ \\
Calcium, g/dl & $9.3 \pm 2.8$ \\
Phosphate, g/dl & $6.1 \pm 2.8$ \\
Parathyroid hormone, pmol/l & $376 \pm 766$ \\
SBP, mm Hg & $152 \pm 26$ \\
DBP, mm Hg & $79 \pm 14$ \\
PP, mm Hg & $72 \pm 17$ \\
\hline
\end{tabular}

used due to the absence of better markers, such as C-reactive protein, interleukins, or fibrinogen, in the data.

We studied the relationship of SBP, DBP, and PP with all-cause mortality, with the aim to identify the best measure. The relationships were tested in unadjusted, adjusted, and bivariate models. The bivariate models were used to determine the effect of each of the three blood pressure variables adjusted for the two others.

Survival analyses were performed with high and low PP measures (above and below 80 $\mathrm{mm} \mathrm{Hg}$ ), albumin values (above and below $3.5 \mathrm{~g} / \mathrm{dl}$ ), white blood cell counts (above and below $10 \times 10^{9} / \mathrm{l}$ ), and presence and absence of diabetes to estimate the unadjusted effect of these markers on mortality. These values were predetermined before analysis based on laboratory reporting of normal values; for example, a white cell count of $10 \times 109 / 1$ or higher and serum albumin less than 3.5 will be considered as abnormal.

The effect of increased PP on mortality was determined for the entire population and then separately in the presence and absence of markers associated with inflammation.

All statistical analyses were performed using SAS 9.1 (Cary, N.C., USA). Institutional approval to perform this study (as part of the operations of the Cardiovascular Special Studies Center of the USRDS) was obtained from the Hennepin County Medical Center/Minneapolis Medical Research Foundation Human Subjects Committee.

\section{Results}

The baseline characteristics of the study population (table 1) were similar to the characteristics of the national cohort of dialysis patients at the time of data collection.

\section{Effect of Baseline Variables on Mortality: Multivariable Analysis}

The initial multivariate analysis with SBP and DBP, adjusted for age, diabetes, haemoglobin, albumin, white blood count, calcium, phosphorus, and parathyroid hormone, showed no relationship of mortality with SBP and a negative relationship of mortality with DBP (table 2). However, PP, adjusted for SBP and all other variables, was associated with increased hazards of mortality [adjusted hazard ratio, PP 1.006 (95\% confidence interval, CI, 1.0021.010); SBP 0.993 (95\% CI 0.990-0.996)]. Age and diabetes were strongly associated with mortality in both analyses. 
Table 2. Proportional hazard regression: association of variables with all-cause mortality in haemodialysis patients

Banerjee et al.: Pulse Pressure and Inflammation in Haemodialysis

\begin{tabular}{llr}
\hline Variable & $\begin{array}{l}\text { Hazard ratio } \\
(95 \% \mathrm{CI})\end{array}$ & $\mathrm{p}$ \\
\hline Age & $1.037(1.034-1.040)$ & $<0.0001$ \\
Diabetes & $1.236(1.147-1.333)$ & $<0.0001$ \\
Haemoglobin & $0.977(0.958-0.995)$ & 0.0136 \\
Albumin & $0.667(0.667-0.727)$ & $<0.0001$ \\
Cholesterol & $0.998(0.997-0.999)$ & $<0.0001$ \\
White blood count & $1.005(1.000-1.010)$ & 0.0355 \\
Phosphate & $1.017(1.007-1.027)$ & 0.0011 \\
Calcium & $1.003(0.990-1.016)$ & 0.6571 \\
Parathyroid hormone & $1.000(1.000-1.000)$ & 0.5546 \\
Coronary heart disease & $1.129(1.041-1.224)$ & 0.0033 \\
Peripheral vascular disease & $1.384(1.267-1.512)$ & $<0.0001$ \\
Cerebrovascular disease & $1.266(1.145-1.400)$ & $<0.0001$ \\
Congestive heart failure & $1.330(1.231-1.437)$ & $<0.0001$ \\
SBP & $0.999(0.996-1.001)$ & 0.2593 \\
DBP & $0.995(0.991-0.999)$ & 0.0220 \\
\hline
\end{tabular}

Table 3. Dual models: PP and SBP, $\mathrm{PP}$ and $\mathrm{DBP}$, and SBP and DBP

\begin{tabular}{cl}
\hline Variables & $\begin{array}{l}\text { Mortality hazard ratio } \\
(95 \% \mathrm{CI})\end{array}$ \\
\hline $\begin{array}{c}\text { Model 1 } \\
\text { PP }\end{array}$ & $1.029(1.027-1.032)$ \\
SBP & $0.981(0.979-0.983)$ \\
Model 2 & $1.010(1.008-1.011)$ \\
PP & $0.981(0.979-0.983)$ \\
DBP & $1.010(1.008-1.011)$ \\
Model 3 & $0.972(0.969-0.974)$ \\
SBP & \\
DBP &
\end{tabular}

Effect of SBP, DBP, and PP on Mortality: Dual Models

With bivariate analyses, PP adjusted for SBP and DBP was positively associated with adverse outcome. In the model with SBP and DBP, increased SBP and decreased DBP were associated with adverse outcomes. PP was again identified as the major determinant of outcome (table 3).

\section{Effects of PP and Markers of Inflammation}

The hazard ratio increases with each $10-\mathrm{mm} \mathrm{Hg}$ rise of $\mathrm{PP}$ adjusted for age, diabetes, and SBP (fig. 1). With Kaplan-Meier survival analysis, a PP $>80 \mathrm{~mm} \mathrm{Hg}$ was associated with increased mortality (fig. 2).

The effect of PP on mortality was attenuated in the presence of the chosen markers of systemic inflammation (white blood count $>10 \times 10^{9} / 1$, albumin $<3.5 \mathrm{~g} / \mathrm{dl}$, diabetes; table 4 ). Mortality hazard ratios increased significantly for each $10-\mathrm{mm} \mathrm{Hg}$ rise of $\mathrm{PP}>70 \mathrm{~mm} \mathrm{Hg}$ with white blood count less than, but not greater than, $10 \times 10^{9} / 1$, from 1.1 at PP $60-69 \mathrm{~mm} \mathrm{Hg}$ to 1.8 at $\mathrm{PP}>110 \mathrm{~mm} \mathrm{Hg}$. With a similar PP rise (from $60-69$ to $>110 \mathrm{~mm} \mathrm{Hg}$ ) in the absence of diabetes, the hazard ratios for mortality increased from 1.1 to 2.2 ; with albumin $>3.5 \mathrm{~g} / \mathrm{dl}$, the hazard ratios increased from 1.1 to 1.8. However, with similar PP changes (from 60-69 to $>110$ 
Table 4. Effect of markers of systemic inflammation on the relationship of PP and mortality

\begin{tabular}{|c|c|c|c|c|c|c|}
\hline \multirow{2}{*}{$\begin{array}{l}\mathrm{PP} \\
\mathrm{mm} \mathrm{Hg}\end{array}$} & \multicolumn{6}{|c|}{ Mortality hazard ratio $(95 \% \mathrm{CI})$} \\
\hline & no diabetes & $\begin{array}{l}\text { white blood count } \\
<10 \times 10^{9} / 1\end{array}$ & $\begin{array}{l}\text { albumin } \\
>3.5 \mathrm{~g} / \mathrm{dl}\end{array}$ & diabetes & $\begin{array}{l}\text { white blood count } \\
>10 \times 10^{9} / 1\end{array}$ & $\begin{array}{l}\text { albumin } \\
<3.5 \mathrm{~g} / \mathrm{dl}\end{array}$ \\
\hline$<50$ & $0.976(0.859-1.109)$ & $0.940(0.842-1.050)$ & $0.899(0.790-1.023)$ & $0.992(0.825-1.192)$ & $1.028(0.773-1.366)$ & $1.018(0.856-1.211)$ \\
\hline $50-59$ & 1.000 & 1.000 & 1.000 & 1.000 & 1.000 & 1.000 \\
\hline $60-69$ & $1.113(1.005-1.232)$ & $1.111(1.021-1.208)$ & $1.106(1.007-1.215)$ & $0.930(0.819-1.055)$ & $0.889(0.714-1.108)$ & $1.055(0.914-1.217)$ \\
\hline $70-79$ & $1.279(1.156-1.414)^{*}$ & $1.191(1.097-1.293)^{*}$ & $1.193(1.089-1.308)^{*}$ & $0.953(0.845-1.076)$ & $1.210(0.916-1.489)$ & $1.246(1.086-1.430)$ \\
\hline $80-89$ & $1.484(1.326-1.660)^{*}$ & $1.375(1.259-1.501)^{*}$ & $1.418(1.286-1.563)^{*}$ & $0.998(0.881-1.138)$ & $1.138(0.916-1.413)$ & $1.205(1.040-1.396)$ \\
\hline $90-99$ & $1.608(1.403-1.844)^{*}$ & $1.406(1.268-1.559)^{*}$ & $1.457(1.301-1.633)^{*}$ & $0.931(0.808-1.071)$ & $1.039(0.808-1.336)$ & $1.154(0.967-1.377)$ \\
\hline $100-109$ & $2.034(1.695-2.440)^{*}$ & $1.583(1.387-1.805)^{*}$ & $1.702(1.472-1.968)^{*}$ & $1.021(0.862-1.210)$ & $1.460(1.076-1.981)$ & $1.323(1.065-1.644)$ \\
\hline$>110$ & $2.231(1.711-2.908)^{*}$ & $1.806(1.495-2.180)^{*}$ & $1.765(1.444-2.157)^{*}$ & $1.155(0921-1.448)$ & $1.397(0.951-2.052)$ & $1.812(1.323-2.483)$ \\
\hline
\end{tabular}

Fig. 1. Hazard ratios for mortality for each $10-\mathrm{mm}$ $\mathrm{Hg}$ rise of $\mathrm{PP}$ adjusted for age, diabetes, and SBP.
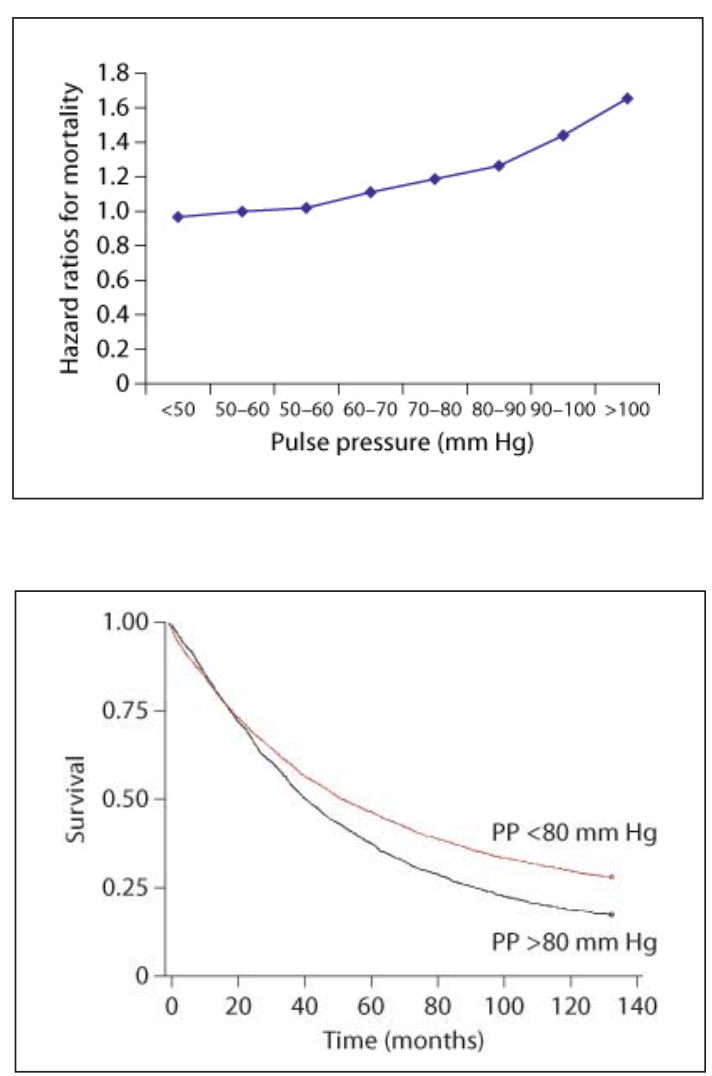

Fig. 2. Survival for patients with $\mathrm{PP}$ above and below $80 \mathrm{~mm} \mathrm{Hg}$.

$\mathrm{mm} \mathrm{Hg}$ ), the changes in hazard ratios were minimal and not statistically significant in the presence of diabetes and low serum albumin. This apparent 'nullification' of PP as an independent predictor of mortality was most evident with regard to diabetes. For example, in nondiabetic ('non-inflamed') patients with PP 90-99 $\mathrm{mm} \mathrm{Hg}$ (compared to 60-69 $\mathrm{mm} \mathrm{Hg}$ ), the hazard ratio was 1.61 (95\% CI 1.40-1.84), versus 0.93 (95\% CI 0.81-1.07) in diabetic patients. In patients with comparable PP (90-99 $\mathrm{mm} \mathrm{Hg})$, the hazard ratio for those with lower white blood cell count ('non-inflamed') was 1.41 (95\% CI 1.27-1.56), versus 1.04 (95\% CI 0.81-1.37) for patients with higher white blood cell count; the hazard ratio for patients with higher serum albumin was 1.46 (95\% CI 1.30-1.63), versus 1.15 (95\% CI 0.97-1.38) for patients with lower 
serum albumin. At the highest PP (>110 $\mathrm{mm} \mathrm{Hg}$ ), there was a two-fold mortality hazard for non-diabetic patients and no significant mortality hazard for diabetic patients. In contrast, a significant mortality hazard was present for patients with this markedly elevated PP irrespective of serum albumin or white blood count, suggesting a possible qualitative difference in diabetes compared with the other inflammatory markers, particularly in patients with severe atherosclerotic disease (as manifested by the markedly abnormal PP).

\section{Discussion}

This study aimed to establish the role of PP on mortality in a large cohort of haemodialysis patients. Interestingly, we found that PP loses its effect on mortality in the presence of systemic inflammation.

We found that PP appears to be a better predictor of adverse outcomes than SBP in patients on maintenance haemodialysis. As expected, DBP was shown to be a negative predictor of mortality. The findings correlate with large studies of US and Japanese haemodialysis patients $[3,4]$. This study thus reinforces the importance of arterial stiffness as a marker of adverse outcomes, as shown in a French haemodialysis population, and PP as a clinical marker of central arterial stiffness [6,7].

We examined the effect of a non-traditional risk factor, systemic inflammation, on the relationship of PP with mortality, as systemic inflammation has recently been proposed to be a significant marker of vascular ill health and adverse outcomes in patients with kidney disease [8]. Systemic inflammation is related to atherosclerosis and hence to increased cardiovascular event rates. We hypothesised that this effect of systemic inflammation on cardiovascular events and cardiovascular mortality would be stronger than the effect of decreased vascular compliance.

Our study found that the effect of PP on mortality was nullified by rising markers of systemic inflammation. Though PP exhibits an effect on mortality due to arterial stiffness, in the presence of markers of inflammation, namely high white blood cell count, low albumin, and diabetes, this effect is lost as the ill effects of systemic inflammation become overwhelming. High white cell count is a crude but well-established marker of systemic inflammation. White blood cell count is perhaps less sensitive than C-reactive protein, interleukins, or fibrinogen; however, in our large dialysis cohort we believe that its clinical significance is important. White cell count as a marker of inflammation has been proven to be a predictor of cardiovascular disease [9]. A study of haemodialysis patients showed a relationship between white blood cell count and C-reactive protein over time [10]. In the same study, serum albumin levels steadily improved with decreasing C-reactive protein [10]. This evidence suggests that systemic inflammation is associated with low albumin as part of a negative acutephase reaction. Diabetes is now recognised to be associated with low-grade inflammation related to its pathogenesis and as a cause of target organ injury. Inflammatory pathways impede insulin activity and predispose to diabetes [11]. However, diabetes can affect vascular function in several pathways, and some of these are independent of inflammation.

The adverse effects of systemic inflammation could be related to both atherosclerotic and non-atherosclerotic cardiovascular diseases. In the general population, inflammation promotes atherosclerosis by causing endothelial dysfunction. Endothelial dysfunction is present and associated with inflammation in patients on haemodialysis. Cardiac fibrosis due to local inflammation in the heart could be another mechanism related to systemic inflammation. It is well known that sudden cardiac death related to cardiomyopathy and arrhythmia is a common cause of death in patients on haemodialysis [12]. Evidence from observational studies suggests an increase in sudden cardiac death with rising C-reactive protein [13]. 
Our study has limitations. As mentioned above, the lack of information on C-reactive protein, interleukins, or fibrinogen as markers of inflammation is a definite disadvantage. White cell count has been used in other studies but is a less sensitive marker of inflammation, and serum albumin is a negative acute-phase reactant but could decrease due to malfunctioning of the liver. Diabetes-related target organ injury is associated with low-grade inflammation, but not always. Due to the nature of the study data, which are from a large database, we also lacked information on a linear relationship between PP and mortality. The study was retrospective and hence there are limitations as regards to information on the change of risk factor profile with time. The changes in the risk factor profile with time can change the outcome significantly. In addition, lack of echocardiographic data on ejection fraction limits the interpretation of systolic function and its relationship with PP and mortality. The extent of atherosclerosis is unknown and hence cannot be related to either traditional or non-traditional risk factors. The white cell count and serum albumin may change with time, and our study measured it at baseline only. This limits accurate assessment of the effects of such measures on the outcome.

However, the study generates an important hypothesis regarding the effect of non-traditional risk factors on the relationship of mortality and traditional risk factors in patients on maintenance haemodialysis. Traditional risk factors cause vascular damage in early chronic kidney disease and before dialysis initiation. The contribution of inflammation to cardiovascular damage may be slight at this stage of chronic kidney disease. Once the inflammation has progressed after dialysis initiation, its effect on endothelial dysfunction, atherosclerosis, and cardiomyopathy may become overwhelming and hence traditional risk factors become less significant. Such a hypothesis needs to be proven with prospective follow-up studies, preferably with intervention.

\section{Acknowledgements}

The Cardiovascular Special Studies Center of the United States Renal Data System is supported by Contract No. HHSN2672007105003C (National Institute of Diabetes and Digestive and Kidney Diseases, National Institute of Health, Bethesda, Md., USA). This study was performed as a deliverable. The data reported here have been supplied by the United States Renal Data System. The authors wish to thank United States Renal Data System colleagues Shane Nygaard for manuscript preparation and Nan Booth, MSW, MPH, for manuscript editing.

\section{Disclosure Statement}

The authors have no conflicts of interest with the subject matter of the manuscript.

\section{References}

1 Herzog CA: How to manage the renal patient with coronary heart disease: the agony and the ecstasy of opinion-based medicine. J Am Soc Nephrol 2003;14:2556-2572.

2 Blacher J, Guerin AP, Pannier B, Marchais SJ, Safar ME, London GM: Impact of aortic stiffness on survival in end-stage renal disease. Circulation 1999;99:2434-2439.

3 Klassen PS, Lowrie EG, Reddan DN, DeLong ER, Coladonato JA, Szczech LA, Lazarus JM, Owen WF Jr: Association between pulse pressure and mortality in patients undergoing maintenance hemodialysis. JAMA 2002;287:1548-1555. 
4 Tozawa M, Iseki K, Iseki C, Takishita S: Pulse pressure and risk of total mortality and cardiovascular events in patients on chronic hemodialysis. Kidney Int 2002;61:717-726.

5 Korevaar JC, van Manen JG, Dekker FW, de Waart DR, Boeschoten EW, Krediet RT: Effect of an increase in C-reactive protein level during a hemodialysis session on mortality. J Am Soc Nephrol 2004; 15:2916-2922.

6 Blacher J, Asmar R, Djane S, London GM, Safar ME: Aortic pulse wave velocity as a marker of cardiovascular risk in hypertensive patients. Hypertension 1999;33:1111-1117.

7 Guerin AP, Blacher J, Pannier B, Marchais SJ, Safar ME, London GM: Impact of aortic stiffness attenuation on survival of patients in end-stage renal failure. Circulation 2001;103:987-992.

8 Schiffrin EL, Lipman ML, Mann JF: Chronic kidney disease: effects on the cardiovascular system. Circulation 2007;116:85-97.

9 Roy D, Quiles J, Avanzas P, rroyo-Espliguero R, Sinha M, Kaski JC: A comparative study of markers of inflammation for the assessment of cardiovascular risk in patients presenting to the emergency department with acute chest pain suggestive of acute coronary syndrome. Int J Cardiol 2006;109: $317-321$.

10 Bossola M, Sanguinetti M, Scribano D, Zuppi C, Giungi S, Luciani G, Torelli R, Posteraro B, Fadda G, Tazza L: Circulating bacterial-derived DNA fragments and markers of inflammation in chronic hemodialysis patients. Clin J Am Soc Nephrol 2009;4:379-385.

11 Wellen KE, Hotamisligil GS: Inflammation, stress, and diabetes. J Clin Invest 2005;115:1111-1119.

12 Port FK, Hulbert-Shearon TE, Wolfe RA, Bloembergen WE, Golper TA, Agodoa LY, Young EW: Predialysis blood pressure and mortality risk in a national sample of maintenance hemodialysis patients. Am J Kidney Dis 1999;33:507-517.

13 Parekh RS, Plantinga LC, Kao WH, Meoni LA, Jaar BG, Fink NE, Powe NR, Coresh J, Klag MJ: The association of sudden cardiac death with inflammation and other traditional risk factors. Kidney Int 2008; 74:1335-1342. 